\title{
Stress among pediatric oncology staff. A systematic review
}

\author{
Maria Zarenti ${ }^{1}$, Evangelia Kressou ${ }^{1}$, Zacharo Panagopoulou ${ }^{1}$, Flora Bacopoulou ${ }^{2}{ }^{\circledR}$, loulia Kokka ${ }^{1}$, Dimitrios \\ Vlachakis $^{2,3,4}$, George P. Chrousos ${ }^{2}$, Christina Darviri ${ }^{1}$
}

'Postgraduate Course of Science of Stress and Health Promotion, School of Medicine, National and Kapodistrian University of Athens, Athens, Greece

${ }^{2}$ University Research Institute of Maternal and Child Health \& Precision Medicine and UNESCO Chair on Adolescent Health Care, National and Kapodistrian University of Athens, Aghia Sophia Children's Hospital, Athens, Greece

${ }^{3}$ Laboratory of Genetics, Department of Biotechnology, School of Applied Biology and Biotechnology, Agricultural University of Athens, Athens, Greece

${ }^{4}$ Lab of Molecular Endocrinology, Center of Clinical, Experimental Surgery and Translational Research, Biomedical Research Foundation of the Academy of Athens, Athens, Greece

Competing interests: MZ none; EK none; ZP none; FB none; IK none; DV none; GPC none; CD none

\section{Abstract}

Cancer is considered one of the dominant life-threatening diseases in children. Working in the field of pediatric oncology, although rewarding, can be a source of stress and emotional burden for health care providers. The aim of this systematic review was to summarise the evidence regarding the occupational stress of health care providers working with pediatric cancer patients. Extensive search of the Pubmed and Scopus databases was performed to identify studies relevant to the topic. Initial search retrieved 657 studies. The reviewing investigators, after applying the inclusion/exclusion criteria, extracted data to critically appraise the quality of evidence. The final step of search concluded in 23 studies of heterogeneous design. Results revealed two main domains of which occupational stress derived from i) the interaction of the health care provider with the patient and the family, and ii) several organisational factors, such as hierarchical structures, experience, workload, and low organisational support. Literature on the stress of pediatric oncology staff is limited. The rather small sample sizes of the studies, the heterogeneity of methodological design, the lack of assessment from a sociological point of view, as well as the limited psychometric instruments adapted to pediatric oncology staff, make the validity of the results questionable. Further research is warranted to obtain a more accurate view of the field, to identify a causeeffect relation between work-related stress and pediatric oncology staff, and, more importantly, to guide future recommendations on support systems and stress management training within pediatric oncology settings.

\section{Introduction}

Cancer is considered one of the dominant lifethreatening diseases in children. According to the World Health Organization, 300,000 new cases are recorded annually up to the age of 19 years (WHO,2020). Working in the field of pediatric oncology and providing psychological and medical assistance in a hospitalsetting can be a rewarding experience. Nevertheless, it can simultaneously be a source of stress and emotional burden for health care practitioners. An intimate interaction between children, parents and pediatric oncology staff is usually developed as part of the hospitalization process. Health care professionals are obliged to deal with the pain of both children and parents (Dilek et al., 2019) due to the frustration deriving from ineffective treatments
(Himelstein et al., 2004). Most studies on the occupational stress of oncology practitioners have focused on nurses, mainly because they are those who appear to have the most frequent and intense relationship with children compared to other staff members. Even though a child's condition may not be lethal, solely the fear of death experienced by the family and the child, is an additive stressful factor that nurses are expected to manage. A nurse becomes a personal brace, a care provider, and a family supporter onadaily basis. The multiple roles increase the risk of distress, fatigue, grief and burnout. Emotional distress for pediatric oncology nurses can be provoked by the additional requirements they need to meet, which are often off the job description, the different parental perspectives regarding the illness, and the lack or insufficiency of coping mechanisms. Research has

\section{Article history}

Received: 1 December 2020

Accepted: 4 Febraury 2021

Published: 22 October 2021

(c) 2021 Zarenti et al.; the authors have retained copyright and granted the Journal right of first publication; the work has been simultaneously released under a Creative Commons Attribution Licence, which allows others to share the work, while acknowledging the original authorship and initial publication in this Journal. The full licence notice is available at http://journal.embnet.org. 
shown that two-thirds of nurses do not feel comfortable providing psychological support to families of dying children (Plante and Cyr, 2011), while in another study nurses found communication with the patient's familyas one of the hardest skills to master (Pearson, 2013). Moreover, health care professionals in oncology settings have reported feelings of despair and disturbance as they felt unable to treat the pain of the young patients (Ergün et al., 2005). Under these circumstances, stress is inevitable and may interfere with the professionals' work performance. According to Lazarus and Folkman, workrelated stress is considered as a physical and emotional reaction to conditions in which the employee's abilities, resources, or needs are inadequate to meet the work demands (Lazarus and Folkman,1984).

Taking these findings into consideration, the aim of this review was to summarise the literature regarding the most common, high-risk factors that might have caused stress in pediatric oncology health care providers.

\section{Materials, Methodologies and Techniques}

\section{Study selection}

A comprehensive search of the Pubmed and Scopus databases was conducted to identify research papers relevant to the examined topic. The following search terms were used in each database: "pediatric oncology" OR "paediatric oncology" AND "job stress" OR "work related stress" OR "occupational stress". All search terms were investigated in titles, abstracts and keywords.

\section{Inclusion and exclusion criteria}

Studies were eligible for inclusion if published in peer reviewed journals, only in the English language. Studies had to focus on the occupational stress of health care providers solely of pediatric cancer patients, regardless of their profession (e.g., medical or non-medical staff). Other relevant reviews or meta-analyses were not included. Research protocols that did not provide sufficient data were also excluded. Studies focusing on burnout syndrome and not on occupational stress were also excluded from the study.

\section{Results}

\section{Basic characteristics of included studies}

Initial search yielded 657 studies. After applying the inclusion/exclusion criteria 23 studies were identified and included in this review. The full screening process is illustrated in Figure 1. Data extracted from each study included the first author and year of publication, sample's characteristics, and main results on work-related stress. The characteristics of the included studies are outlined in Table 1 in Supplementary Data ${ }^{1}$.

${ }^{1}$ http://journal.embnet.org/index.php/embnetjournal/article/ downloadSuppFile/981/981_supp_1

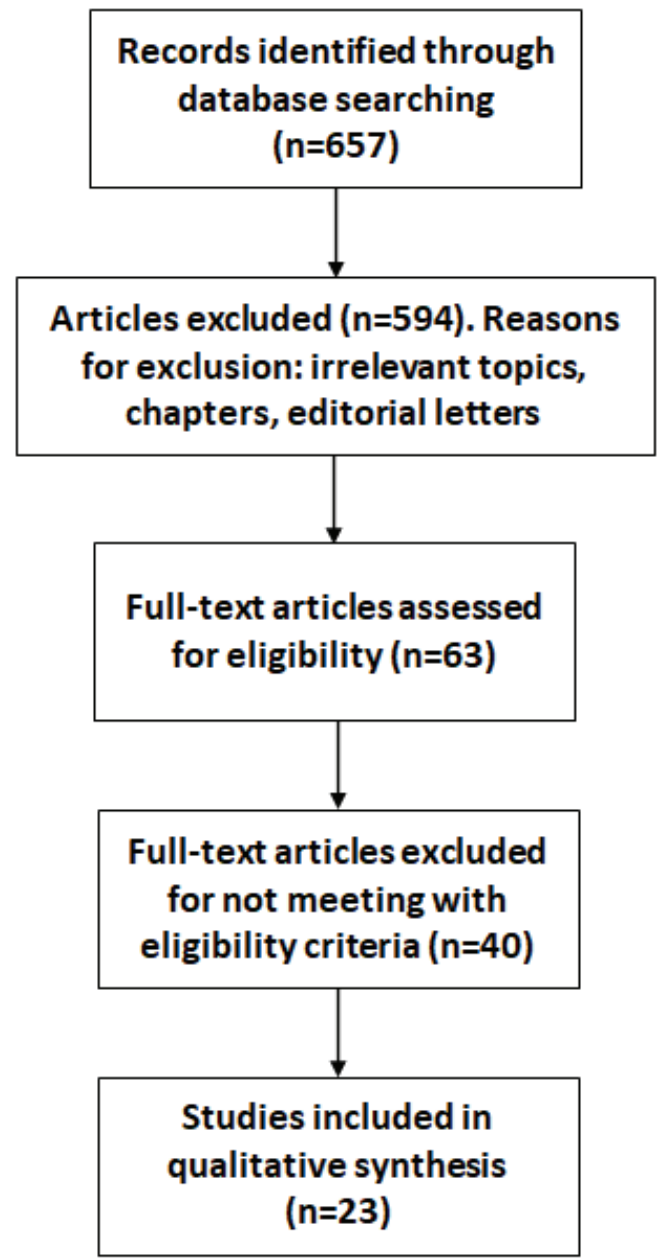

Figure 1. Flow chart of the studies included in the review.

\section{Main Findings}

In the study of Kpassagou and Soedje, health practitioners demonstrated high levels of emotional distress which derived from the lack of qualitative medical equipment (Kpassagou and Soedje, 2017). In addition, McCloskeyand Taggart highlighted the lack of services, funding and high administration responsibilities, as work-related stress factors (McCloskey and Taggart, 2010). Less experienced nurses have mentioned the lack of consideration by physicians, unit management, and parents as additional stressful factors (Morrison and Morris, 2017), while staff support interventions have appeared available but mostly for doctors compared to nurses (Beresfordet al., 2018). Team conflict, bad functioning, and feeling of not being valued by the organization were identified as main stressors (McCloskey and Taggart, 2010; Taylor and Aldridge, 2017; Ostadhashemi et al., 2019; Beresford et al., 2018). In addition, Gallagher and Gormley noted that bone marrow transplant (BMT) nurses experienced lower levels of stress related to the patients'critical condition as years of professional experience increased (Gallagher and Gormley, 2009). Pediatric BMT nurses have also reportedrotating shiftsand short staffing as sources of stress with long working hours as the least 
stressful factor (McCloskey and Taggart, 2010; Gallagher and Gormley, 2009; Hinds, 2000). Stenmarker et al. identified as oncologists' stressful factors tasks affecting their leisure time (31.1\%), economic discussions (15.5\%) and organizational aspects at the place of work (36.6\%) (Stenmarker et al., 2009). In another study, oncologists reported both workload and lack of time as their main work burden. However, pediatric oncologists in this study demonstrated low levels of emotional distress (Stenmarker et al.,2009). Taylor et al. identified as a key stressor the unfamiliarity with a specific treatment for children (Taylor and Aldridge, 2017). Furthermore, social workers' experiences in another study, indicated that a combination of lack of professional competence, low organizational support and professional inferiority caused stress and personal exhaustion (Pergert et al., 2019).

According to Chang et al., nurses noted that 'making mistakes' had the highest mean score in the stressor scale for pediatric oncology nurses (SSPON) despite the availability of clinical support nurses (CSNs). Also, nurses without CSNs pointed higher levels of work-related stress (Changet al., 2007). This finding was supported by another study where no stress management training was provided (Kpassagou and Soedje, 2017). Another research demonstrated that higher levels of organizational commitment were associated with higher perception of role-related stressors. Also, the level of expertise and different levels of education in nursing were identified as factors affecting stress levels (Hinds et al., 2003). Stress levels seem to be further elevated by increased levels of uncertainty (Af Sandeberg et al., 2020). Mukherjee et al., in a validation study of two psychometric tools, the Work Stressors Scale - Paediatric Oncology (WSSPO) and the Work Rewards Scale - Paediatric Oncology (WRS-PO) have shown that the sources of work-related stress were multi-dimensional and included general team-centered/organizational stressors (e.g. workload, team conflict) (Mukherjee et al., 2014).

Kpassagou and Soedje found that health practitioners experienced high levels of emotional distress which derived from unpredicted treatment failure. Additional sources of emotional distress comprise delivery of bad news to the patients' family (Kpassagou and Soedje, 2017; Bowden et al., 2015; Cline et al., 2020), as well as informing adolescents about their disease (Stenmarker et al.,2009). In cases where recovery is not possible, nurses may be asked by the parents to withhold the truth from their child, which is reported to be a cause of great distress (Hopia and Heino-Tolonen, 2019). Another study reported multiple sources of nurses' stress i.e., multiple caregiver roles (e.g., medical, emotional and spiritual care, therapeutic relationships, setting boundaries) (Morrison and Morris, 2017; McCloskey and Taggart, 2010; Taylor and Aldridge, 2017). According to Klassen et al. some health care providers described communication problems/conflicts with the patients' parents (e.g., family refuses to stop treatment) as additional stressors (Klassen et al., 2012). On the other hand, pediatric nurses experienced distress derived from the lack of time to build a close relationship with the family (McCloskey and Taggart, 2010). Papadatou et al., identified additional sources of stress with the use of a scale with the most and least stressful conditions as perceived by oncologists and nurses. The participants organised, in hierarchical order, the conditions of distress (Papadatou et al., 2002). Caring for a dying child and witnessing pain were considered the most stressful conditions (McCloskey and Taggart 2010; Chang, 2007; Papadatou et al., 2002; Hinds, 2000). For both the doctors and nurses, the unexpected death of a patient was considered as a highly stressful event. Half of the nurses were more distressed than the physicians because of their longstanding relationship with the children (Papadatou et al., 2002). The intimate relationship built with children and families was addressed asboth stressful and rewarding (Taylor and Aldridge, 2017; Bowden et al., 2015), while being the bearer of "bad news" was found to be very stressful amongst translators for families of different nationalities (Cline et al., 2020). Furthermore, nurses rated as less stressful losing the patient at home rather than in the hospital (De Carvalho et al., 2005). In another study, pediatric BMT nurses ranked the three top work-related stressors in order of importance and the most stressful factor was critical illness of the patients (Gallagher and Gormley, 2009). Furthermore, nurses reported demanding families and dying patients as sources of stress (Gallagher and Gormley, 2009; Bowden et al., 2015).

Provision of palliative care seemed to be a burden for physical and mental health, causing repetitive distressing dreams (Fanos, 2007) and high levels of somatization (Stenmarker et al., 2009). According to some studies, job satisfaction, life satisfaction, high sense of coherence, social support and perceived effectiveness seemed to mediate stress responses (Stenmarker et al., 2009; Rheingans, 2008; Hinds et al., 2003).

The importance of a parents' capability and resources to support the upbringing and development of their children during hospitalization has been addressed by Hopia and Heino-Tolonen. According to their study, the lack of resources prevented some parents from spending enough time with their children and resulted in feelings of loneliness for the child. These situations generated stress and feelings of sadness in the nursing staff as well (Hopia and Heino-Tolonen, 2019).

\section{Discussion}

In this review two domains of work-related stress among pediatric oncology staff were identified. The first domain included organizational issues such as workload and time pressure, and communication with colleagues. Specifically, health care providers were affected by the lack of colleagues' consideration, medical supplies, medical staff, and the fear of making mistakes. The second domain included interactions of the health care provider with the patient and the family i.e., provision of 
palliative care, conflicting points of view about necessary patient care, communication with patients' families, multiple responsibilities, witnessing pain and patient loss. Although most health care providers experienced high levels of stress, no support systems were provided by the hospitals' administration systems. Health care practitioners in the study of Kpassagou and Soedje reported increased emotional distress due to the lack of training in coping with loss of a life and prolonged patient suffering (Kpassagou and Soedje, 2017). Even in cases where support was provided, only one study concluded that health care providers benefited from it with respect to their stress levels (Chang et al., 2007).

Literature on stress of pediatric oncology staff up to date is limited. The relatively small sample sizes, as well as the limited psychometric instruments adapted to pediatric oncology staff, make the results' validity questionable. Furthermore, most quantitative studies did not apply the same measurements. In addition, the heterogeneous methodological approaches with respect to study design cannot conclude in results with certainty. Worth mentioning is the fact that the cross-sectional design of some of the studies cannot investigate causeeffect relationship, and mediating factors may have affected the results.

Further research is needed to establish the causeeffect relation between work-related stress and pediatric oncology staff. Future recommendations should include support systems and stress management training within hospitals especially to pediatric oncology staff exposed to highly stressful circumstances. Policy makers should develop and implement interventional programs on the management of pediatric oncology workers' stress. Common interventions implemented to increase nurse's resilience include workshops, focus groups, mindfulness techniques and educational seminars regarding the effects of stress and possible ways of its management (Boyle and Bush, 2018). Concerns have been raised regarding the psychological support that doctors and nurses need (Beresford et al., 2018). This is on some degree questionable, since research demonstrated that nurses and not doctors are those who are mostly exposed to work-related stress. Finally, a factor that should be considered while developing prevention programs in pediatric oncology settings, is that of accessible clinical support by all professionals. This is of high importance due to cultural differences among doctors and nurses, with the former appearing in less need for support as they appear more "resilient" and distant (Hopia and Heino-Tolonen, 2019). Careful development of effective intervention programs for stress management of

\section{Key Points}

- Working in pediatric oncology settings can be a source of stress for health professionals.

- Organisational issues, interaction with families and coping with illness and death are among the most stressful factors.

- Nurses experience higher stress levels compared to other health professions dealing with pediatric cancer.

- Stress management intervention programs for health care providers should be embedded in pediatric oncology settings.
}

pediatric oncology staff could lead to a more sufficient clinical practice and consequently contribute to the amelioration of life quality of hospitalized children suffering from cancer.

\section{References}

1. Af Sandeberg M, Bartholdson C, Pergert P (2020) Important situations that capture moral distress in paediatric oncology. BMC Medical Ethics 21(6), 2-9. http://dx.doi.org/10.1186/ s12910-020-0447-x.

2. Beresford B, Gibson F, Bayliss J, Mukherjee S (2018) Preventing work-related stress among staff working in children's cancer Principal Treatment Centres in the UK: a brief survey of staff support systems and practices. European Journal of Cancer Care 27(2), e12535. http://dx.doi.org/10.1111/ecc.12535.

3. Bowden MJ, Mukherjee S, Williams LK, DeGraves S, Jackson M et al. (2015) Work-related stress and reward: an Australian study of multidisciplinary pediatric oncology healthcare providers. Psycho-Oncology 24(11), 1432-1438. http://dx.doi.org/ 10.1002/. pon.3810.

4. Boyle DA and Bush NJ (2018) Reflections on the emotional hazards of pediatric oncology nursing: four decades of perspectives and potential. Journal of Pediatric Nursing 40, 63-73. http://dx.doi. org/10.1016/j.pedn.2018.03.007.

5. Chang A, Kicis J, Sangha G (2007) Effect of the clinical support nurse role on work-related stress for nurses on an inpatient pediatric oncology unit. Journal of Pediatric Oncology Nursing 24(6), 340-349. http://dx.doi.org/10.1177/1043454207308065.

6. Cline GD, Steinberg DM, Kentor RA, Shellman AB, Fritz AM et al. (2020) Short report: sticks and stones may break my bones, but words can also hurt me: understanding language interpreters' perceptions of stress/ growth and needs in a pediatric medical setting. Psychology, Health \& Medicine 10, 1-9. http://dx.doi.org /10.1080/13548506.2020.1788104.

7. De Carvalho EC, Muller M, De Carvalho PB, de Souza Melo A (2005) Stress in the professional practice of oncology nurses. Cancer Nursing 28(3), 187-192. http://dx.doi. org/10.1097/00002820-200505000-00004.

8. Dilek Konukbay RN, Dilek Yildiz RN, Derya Suluhan RN (2019) Effects of working at the pediatric oncology unit on personal and professional lives of nurses. International Journal of Caring Sciences 12(2), 1-7.

9. Ergun FS, Oran NT, Bender CM (2005) Quality of life of oncology nurses. Cancer Nursing 28(3), 193-199.

10. Fanos JH (2007) "Coming through the fog, coming over the moors": The impact on pediatric oncologists of caring for seriously ill children. Journal of Cancer Education 22(2), 119-123. http://dx.doi.org/10.1007/BF03174360.

11. Gallagher R, Gormley DK (2009) Perceptions of stress, burnout, and support systems in pediatric bone marrow transplantation nursing. Clinical Journal of Oncology Nursing 13, 681-685. http://dx.doi.org/10.1188/09.CJON.681-685.

12. Global Initiative for Childhood Cancer. World Health Organisation. Retrieved July 10th 2020: https://www.who.int/ cancer/childhood-cancer/en/.

13. Himelstein BP, Hilden JM, Boldt AM, Weissman D (2004) Pediatric palliative care. New England Journal of Medicine 350(17), 1752-1762. http://dx.doi.org/10.1056/NEJMra030334.

14. Hinds PS (2000) Testing the stress-response sequence in pediatric oncology nursing. Journal of Pediatric Oncology Nursing 17(2), 59-68. http://dx.doi.org/10.1053/jg.2000.5295.

15. Hinds PS, Srivastava DK, Randall EA, Green A, Stanford D et al. (2003). Testing the revised stress-response sequence model in pediatric oncology nurses. Journal of Pediatric Oncology Nursing 20(5), 213-232. http://dx.doi.org/10.1177/1043454203253785.

16. Hopia A and Heino-Tolonen $\mathrm{T}$ (2019) Families in paediatric oncology nursing: critical incidents from the nurses' perspective. Journal of Pediatric Nursing 44, 28-35. http://dx.doi. org/10.1016/j.pedn.2018.10.013. 
17. Klassen A, Gulati S, Dix D (2012) Health care Providers perspectives about working with parents of children with cancer: a qualitative study. Journal of Pediatric Oncology Nursing 29(2), 92-97. http://dx.doi.org/10.1177/1043454212438405.

18. Kpassagou BL,Soedje KMA (2017) Health practitioners emotional reactions to caring for hospitalized children in Lome, Togo: a qualitative study. BMC Health Services Research 17(2), 700. http://dx.doi.org/10.1186/s12913-017-2646-9.

19. Lazarus RS and Folkman S (1984) Stress appraisal and coping, 1st ed. Springer, New York.

20. McCloskey S and Taggart L (2010) How much compassion have I left? An exploration of occupational stress among children's palliative care nurses. International Journal of Palliative Nursing 16(5), 233-240. http://dx.doi.org/10.12968/ijpn.2010.16.5.48144.

21. Morrison CF and Morris EJ (2017) The practices and meanings of care for nurses working on a pediatric bone marrow transplant unit. Journal of Pediatric Oncology Nursing 34(3), 214-221. http://dx.doi.org/10.1177/1043454216688637.

22. Mukherjee S, Beresford B, Tennant A (2014) Staff burnout in paediatric oncology: new tools to facilitate the development and evaluation of effective interventions. European Journal of Cancer Care 23(4), 450-461. http://dx.doi.org/10.1111/ecc.12176.

23. Ostadhashemi L, Arshi M, Khalvati M, Eghlima M, Khankeh HR (2019) Social workers in pediatric oncology: a qualitative study in Iranian context. Asian Pacific Journal of Cancer Prevention 20(6), 1871-1877. http://dx.doi.org/10.31557/APJCP.2019.20.6.1871.

24. Papadatou D, Bellali T, Papazoglou I, Petraki D (2002) Greek nurse and physician grief as a result of caring for children dying of cancer. Pediatric Nursing 28(4), 345-353.
25. Pearson HN (2013) "You've only got one chance to get it right": children's cancer nurses' experiences of providing palliative care in the acute hospital setting. Issues in Comprehensive Pediatric Nursing 36(3), 188-211. http://dx.doi.org/23758218.

26. Pergert P, Bartholdson C, Blomgren K, af Sandeberg M (2019) Moral distress in paediatric oncology: Contributing factors and group differences. Nursing Ethics 26(7-8), 2351-2363. http:// dx.doi.org/ 10.1177/0969733018809806

27. Plante J and Cyr C (2011) Health care professionals' grief after the death of a child. Paediatrics \& Child Health 16(4), 213-216. $\mathrm{http}: / / \mathrm{dx}$.doi.org/ 10.1093/pch/16.4.213

28. Rheingans JI (2008) Pediatric oncology nurses' management of patients' symptoms. Journal of Pediatric Oncology Nursing 25(6), 303-311. http://dx.doi.org/10.1177/1043454208323294.

29. Stenmarker M, PalmerusK, Marky I (2009a) Life satisfaction of Swedish pediatric oncologists: The role of personality, workrelated aspects, and emotional distress. Pediatric Blood \& Cancer 53(7), 1308-1314. http://dx.doi.org/10.1002/pbc.22251

30. Stenmarker M, Palmerus K, Marky I (2009b) Stress-resilience capacity of pediatric oncologists: A swedish nationwide and population-based study of motivation, emotional distress, and overall life satisfaction. Pediatric Blood \& Cancer 52(4), 503-509. http://dx.doi.org/10.1002/pbc.21849.

31. Taylor $J$ and Aldridge $J$ (2017) Exploring the rewards and challenges of paediatric palliative care work-a qualitative study of a multi-disciplinary children's hospice care team. BMC Palliative Care 16(1), 73. http://dx.doi.org/10.1186/s12904-017-0254-4. 